\title{
Les Jardins de Jacques Delille et le modèle du jardin paysager
}

\section{"Les Jardins" by Jacques Delille and the landscape garden}

\author{
Barbara Łuczak
}

Université Adam Mickiewicz de Poznań

\begin{abstract}
In the article we study the image of the garden in Jacques Delille's well known poem Les Jardins (1782). We argue that the structure of Delille's poetical vision resembles in some aspects the scheme of the 18th century English landscape garden and examine some of its characteristic elements such as: irregular design, a representation of time in space, imitation of nature, the use of landscape painting techniques, etc.
\end{abstract}

Keywords: Jacques Delille, English garden, landscape garden, 18th century didactic poetry, gardens in literature

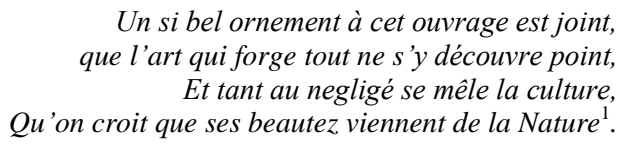

Le Tasse, La Jérusalem délivrée

La seconde partie du XVIII ${ }^{\mathrm{e}}$ siècle est une période durant laquelle sont parus de nombreux poèmes descriptifs de caractère didactique, consacrés aux différents aspects liés au monde végétal : la théorie et la pratique du jardinage, l'évolution saisonnière de la végétation, la succession des labeurs champêtres, les plantes en tant qu'objet de réflexion scientifique, etc. Les Saisons (1769) de Jean-François de Saint-Lambert, Les mois (1779) de Jean-Antoine Roucher, Les Jardins (1782) de Jacques Delille et Les plantes (1797) de René-Richard Castel en sont les exemples représentatifs. Parmi ces textes, celui de Delille occupe une place, d'une certaine manière, privilégiée, étant le plus connu et considéré parfois comme le plus réussi. Cette opinion est partagée par M. J. Chénier qui,

\footnotetext{
${ }^{1}$ Nous conservons l'orthographe originale des passages cités.
} 
dans son Tableau historique de la littérature française, observe l'intérêt des poètes pour les choses de la nature et le place dans le contexte général du développement du poème descriptif et didactique à la fin du XVIII ${ }^{\mathrm{e}}$ siècle et au début du XIX ${ }^{\mathrm{e}}$ siècle :

[Le poème uniquement descriptif] inventé dans les collèges par les poètes latins modernes, embelli par les Anglais, usé par les Allemands, était inconnu parmi nous aux maîtres de la poésie, avant Saint-Lambert et M. Delille. Toutefois, dans les ouvrages de ces deux poètes justement renommés, les défauts essentiels au genre sont rachetés par les beautés nombreuses qui appartiennent à leur génie. Les productions de leurs élèves n'ont pas souvent mérité la même louange. Sans doute, M. Castel, dans le poëme des Fleurs ; M. Lalane, en deux petits poëmes : les Oiseaux de la Ferme, et le Potager; M. Michaud, dans le Printemps d'un proscrit, ont fait preuve de quelque talent pour écrire en vers ; mais savent-ils changer de ton? savent-ils animer la nature ? et les continuelles descriptions qu'ils accumulent avec complaisance, ne fatiguent-elles pas un peu l'attention du lecteur le plus favorablement disposé ? (Chénier, 1824 : 277).

Pour Chénier, les poèmes de Delille et de Saint-Lambert constituent un modèle incontournable, sinon unique, du genre descriptif. Plus loin, Delille, traducteur réputé de Virgile et Milton, "chef d'une école » «dont les défauts brillants sont et seront trop imités, mais dont les beautés, presque sans nombre, auront trop peu d'imitateurs », est reconnu comme l'autorité dans le domaine de «l'éloquence des expressions, le choix des images, et le charme puissant des beaux vers » (Chénier, 1824: 274, 283 et 262), malgré quelques imperfections ou défauts. De l'autre côté, on entend également des voix très critiques des détracteurs de Delille, dont Antoine Rivarol avec sa virulente Lettre critique sur le poème des Jardins $(1782)^{2}$. L'écart entre ces jugements si profondément dissemblables est important (et comme tel pourrait constituer un thème d'analyse à part entière), mais ce qui frappe peut-être plus encore est le fait que ni les admirateurs ni les opposants ne prennent en considération la spécificité thématique des ouvrages étudiés, qu'ils préfèrent aborder par le biais de leur appartenance au «genre descriptif » (Chénier, 1824 : 276). En d'autres termes, si l'on a bien examiné les différents procédés poétiques employés dans ces textes et posé une réflexion sur la place que ceux-ci occupent dans la tradition du poème descriptif, on a en revanche consacré peu d'attention au rapport qui existe entre ces procédés et les propos spécifiques exprimés dans chacun de ces poèmes. Et pourtant, ce rapport reste essentiel pour la construction du sens d'un texte littéraire, de telle sorte que négliger cet élément peut générer des lectures appauvries, arbitraires ou même erronées ${ }^{3}$. C'est la raison pour laquelle, dans

\footnotetext{
${ }^{2}$ Publiée sous le pseudonyme de Barruel (1782).

${ }^{3}$ Par exemple, cette façon d'envisager le problème est peu satisfaisante dans la lecture du poème de Castel, Les plantes. Castel déclare « avoir donné, au commencement du premier Chant,
} 
notre article, plutôt que de revenir sur le débat concernant les aspects stylistiques, métriques ou généalogiques de la poésie du traducteur de l'Énéide, nous voulons mettre en avant l'importance d'un élément qui, à notre avis, n'a pas été suffisamment, ou convenablement, valorisé dans les lectures des Jardins; il s'agit de l'influence du modèle de jardin paysager sur la construction de l'univers poétique de Delille.

Pour atteindre cet objectif, partons d'un fragment du chant I, où Delille présente des règles qui, selon lui, doivent guider les adeptes de l'art du jardinage :

Ce que votre terrein adopte avec plaisirs,

Sachez le reconnoître, osez vous en saisir.

C'est mieux que la nature, et cependant c'est elle ;

C'est un tableau parfait qui n'a point de modèle $(15)^{4}$.

Dans le troisième vers de cette citation, nous trouvons un élément que l'on peut également observer dans le fragment de la Jérusalem délivrée que nous avons adopté comme devise au début de notre article. Selon Mario Praz (1974 : 120), il reflète un premier pas vers l'élaboration du modèle de jardin paysager. En effet, les mots du Tasse s'accordent parfaitement à ceux écrits par un gentleman anglais dans une lettre de 1734, où il caractérise le travail de William Kent, l'un des concepteurs de ce modèle de jardin : « [W]hen finished, it has the apperance of beautiful nature, and without being told, one would imagine art had no part in the finishing » (cit. Richardson, $2008: 291$ ). Delille, quant à lui, ne rejette pas entièrement les principes du jardin régulier, créé, à son avis, « pour briller chez les grands et les rois » (25), mais se déclare partisan du modèle du jardin à l'anglaise : « [M]ais enfin l'Angleterre / Nous apprit l'art d'orner et d'habiller la terre » (48). Par la suite, dans une image bien lisible, le poète compare le

une idée d'importance de la Botanique » (1802: vii) et, plus tard, ne cache pas sa fierté d'être, dans son opinion, le premier à présenter les propos botaniques dignes d'être sujet d'un texte poétique. De cette façon, il peut même déclarer avoir introduit la botanique au Parnasse : "Pour moi qui le premier, sur le mont poétique, / A la cour des neuf Sœurs guidai la botanique » (1803 : 63). Delille, quant à lui, dans l'introduction aux Jardins, rejette directement les propos scientifiques qu'il considère inadéquats dans un poème. Il écrit a propos du traité du père Rapin : « On devine d'avance ce long catalogue et cette énumération fastidieuse qui appartient plus à un botaniste qu'à un poète: et cette marche méthodique, qui feroit un mérite dans un traité en prose, est un grand défaut dans un ouvrage en vers, où l'esprit demande qu'on le mène par des routes un peu détournées, et qu'on lui présente des objets inattendus » (1782 : vii). Par conséquent, les objectifs que Castel poursuit dans son ouvrage consacré aux aspects botaniques de l'art du jardinage sont tellement différents de ceux de Delille que l'approche consistant à juger ces textes l'un par rapport à l'autre, appliquée par Chénier dans son Tableau, devient peu convaincante.

${ }^{4}$ Toutes les citations des Jardins ou l'art d'embellir les paysages de Delille sont issues de sa première édition (voir la liste des références). Le numéro de la page est indiqué entre parenthèses. 
jardin de Le Nôtre au discours bien ficelé d'un orateur dont le charme dure peu et l'abandonne " pour chercher un ami qui [lui] parle du cœur» (27) - l'auteur emprunte ici un vers de Bérénice de Racine -, c'est-à-dire en faveur du jardin irrégulier de Kent.

Cet intérêt pour le jardin à l'anglaise explique le paradoxe que l'on observe dans la manière dont l'auteur procède pour regarder et décrire la nature. Delille conseille à plusieurs reprises de l'imiter: «Pour donner aux jardins une forme plus pure / Observez, connoissez, imitez la nature » (13) ; « Voulez-vous mieux l'orner? Imitez la nature » (49). L'imitation de la nature semble alors une condition du succès du jardinier. Cependant, dans le même chant I, on trouve un passage qui fait réviser cette constatation. La Nature personnifiée s'adresse au Génie en lui parlant de cette manière :

Tu vois tous ces trésors; ces trésors sont à toi.

Dans leur pompe sauvage et leur brute richesse,

Mes travaux imparfaits implorent ton adresse »

Elle dit. Il s'élance [...]

Il retouche en passant le tableau qui varie [...].

Il ne compose pas ; il corrige, il épure,

Il acheve les traits qu'ébaucha la Nature (21-22).

Le paradoxe dont on a fait mention consiste en ce que le poète, d'un côté, affirme que tant lui que ses disciples doivent imiter la nature, et, de l'autre, souligne son insuffisance. Bien qu'elle soit un modèle à suivre, la nature a besoin d'être perfectionnée : «l'inculte énergie» des rocs doit être domptée et sa « sécheresse austère » ornée de la verdure des bois (52). Cette position explique la fréquence avec laquelle le verbe "embellir», présent également dans le sous-titre du poème («L'art d'embellir les paysages »), est utilisé dans le texte. Cette admiration pour le jardin irrégulier, qui transforme le paysage pour augmenter l'illusion de naturalité, rend plus compréhensibles les passages du chant $\mathrm{I}$, où le poète fait une recommandation à ses lecteurs : «Et des champs apprenez l'art de parer les champs » (14), ou, tout en s'adressant aux arbres, dit: «C'est de vous que je veux apprendre à vous orner » (33). En effet, l'idée que l'imitation de la nature peut être supérieure à la nature même constitue le fondement esthétique du jardin paysager anglais du XVIII ${ }^{\mathrm{e}}$ siècle (cf. Richardson, 2008: 8). La tension existant entre ces deux éléments est une force organisatrice de l'univers poétique des Jardins et on peut l'observer tout au long du poème.

Comment corriger la nature pour qu'elle se montre (naturellement) belle ? Comment le Génie devrait-il agir pour que, suite à son intervention, la nature devienne parfaite? Les théoriciens et praticiens du jardin irrégulier répondent à cette question et Delille suit leurs traces. L'œil, qui dans son poème regarde la nature, n'est pas «innocent» (inutile d'ajouter qu'il ne peut pas l'être) et ne le 
prétend pas non plus. La stratégie de regarder la nature - et de la décrire - consiste à la percevoir à travers le prisme de la tradition de ses représentations artistiques : picturales et poétiques. Chez Delille, c'est l'art qui organise et ordonne l'expérience d'observer la nature, mais aussi qui instaure un horizon d'expectatives. Le poète appelle à aimer « des jardins la beauté naturelle » (27), pour, peu après, chercher cette naturalité chez Milton, dans sa description artistique du paradis, où «[s]ans contrainte, sans art, de ses douces prémices / La Nature épuisa les plus pures délices » $(27)^{5}$. Il propose aux adeptes du jardinage de s'inspirer de la peinture paysagiste et, de cette façon, de rendre à la nature ce qui, fruit du travail d'élaboration artistique, paraît « naturel » :

C'est un tableau parfait qui n'a point de modèle.

Ainsi savoient choisir les Berghems, les Poussins.

Voyez, étudiez leurs chef-d'œuvres divins :

Et ce qu'à la campagne emprunta la peinture,

Que l'art reconnoissant le rende à la nature (15-16).

La stratégie appliquée par Delille au moment de regarder la nature fait penser aux observations de Barthes concernant la description :

Toute description littéraire est une vue. On dirait que l'énonciateur, avant de décrire, se poste à la fenêtre, non tellement pour bien voir, mais pour fonder ce qu'il voit par son cadre même : l'embrasure fait le spectacle. Décrire, c'est donc placer le cadre vide que l'auteur réaliste transporte toujours avec lui [...], devant une collection ou un continu d'objets inaccessibles à la parole sans cette opération maniaque $[\ldots]$; pour pouvoir en parler, il faut que l'écrivain, par un rite initial, transforme d'abord le « réel » en objet peint (encadré) ; après quoi il peut décrocher cet objet, le tirer de sa peinture [...]. Ainsi le réalisme $[\ldots]$ consiste, non à copier le réel, mais à copier une copie (peinte) du réel [...] $(1970: 61)^{6}$.

Et Villanueva ajoute : «What realist fiction presents as a real body or object is already a copy of a model articulated by artistic codes and conventions » (Villanueva, 1997 : 43). Effectivement, le modus operandi appliqué par Delille est semblable : il regarde l'espace naturel et le cadre en le transformant en une image à la Poussin ou à la Berghem; de cette façon, il adapte sa vision à un

${ }^{5}$ Dans la traduction du Paradis perdu de Milton, réalisée par Delille, nous lisons: « L'art n'assujettit point leurs [de fleurs] tribus prisonnières ; / La nature, au hasard, d'une prodigue main / de la terre émaillée en a paré le sein » (Delille, $1863: 639$ ).

${ }^{6}$ Dans le contexte de ces constatations de Barthes, l'observation ironique lue chez SainteBeuve, à savoir que la société charmante à laquelle Delille faisait partie "se trouvait alors si bien dans le salon, qu'on mettait au plus la tête à la fenêtre pour voir la nature ; ...et encore, c'était du côté du jardin », prend un sens imprévu (Sainte-Beuve, $1884: 80$ ). 
modèle de «naturalité » codifié et structuré par la tradition des représentations de la nature.

Que Delille cite les noms des maîtres de la peinture paysagiste ne surprend pas, car l'admiration pour les œuvres de Nicolas Poussin, Claude Lorrain et Salvatore Rosa a été un des facteurs décisifs pour la conceptualisation du jardin irrégulier. À cet égard il est intéressant de noter que les toiles de ces artistes ont été trouvées dans l'atelier de Kent, après sa mort (cf. Mayer, 2011 : 17). À cette liste de peintres dont les tableaux ont exercé une influence plus ou moins marquée sur l'art paysagiste au XVIII siècle, notamment en Angleterre, on pourrait ajouter Gaspard Dughet ou bien Jan Frans van Bloemen, entre autres (cf. Richardson, 2007 : 400-402). Delille souligne le rôle que la peinture a joué dans le lancement du nouveau goût : "De nos jardins voués à la monotonie / Leur sublime âpreté jadis étoit bannie. / Depuis qu'enfin le peintre [...] / Sur l'arpenteur timide a repris tous ses droits » (51). Dans ce sens, il n'est pas inutile de rappeler que Kent, au début de sa carrière, a exercé comme peintre et, au cours de son séjour à Rome, a pu se familiariser avec la peinture de Poussin, Lorrain et Rosa (cf. De Bruyn, 2001: 137). Horace Walpole, dans son essai On Modern Gardening, est d'avis que la formation artistique de Kent a joué un rôle déterminant dans la formation de son œuvre :

At that moment appeared Kent, painter enough to taste the charms of landscape. [...] [T] he pencil of his imagination bestowed all the arts of landscape on the scenes he handled. The great principles on which he worked were perspective, and light and shade. $[\ldots][\mathrm{H}] \mathrm{e}$ realised the compositions of the greatest masters in painting » (Walpole, $1798: 536)$.

D'autre part, il ne faut pas oublier que Kent se consacrait également au dessin des intérieurs, ce qui n'est pas anodin pour notre sujet, car la séquence d'espaces disposés en enfilade, une configuration de pièces typique d'une maison, est devenue l'un des éléments clés de sa conception du paysage (cf. Richardson, 2008 : 271) et, par conséquent, de la vision poétique du jardin chez Delille.

Dans le texte de Delille, l'idée du jardin anglais se manifeste alors à différents niveaux. D'abord, par l'évocation directe de la figure de Kent. Même si le poète ne cite son nom qu'une seule fois et le place juste à côté de celui de Le Nôtre - et apparemment sans déclarer sa préférence pour l'un ou l'autre ( « Je ne décide point entre Kent et Le Nôtre », 25) -, son choix se fait évident à mesure qu'il chante la «beauté naturelle» des jardins. De plus, dans une note qui accompagne le texte du poème, Kent est présenté comme "architecte et dessinateur fameux en Angleterre, [...] le premier qui tenta avec succès le genre libre qui commence à se répandre dans toute l'Europe » (97). Visiblement, pour Delille, le rôle de pionnier que Kent a joué en matière d'élaboration du modèle irrégulier ne fait aucun doute, même s'il connaît d'autres jardins anglais de 
l'époque, par exemple, celui de Twickenham ${ }^{7}$, propriété du poète Alexander Pope, qui a occupé, lui aussi, une place importante dans l'histoire du jardinage paysagiste $^{8}$.

Deuxièmement, la prétendue naturalité du jardin à l'anglaise et la disposition spontanée de ses éléments se devinent dans la structure générale du texte de Delille. L'auteur signale cet aspect dans l'introduction de la première édition des Jardins, où il oppose la structure de son poème à la composition des Libri Hortorum IV de Rapin. Ce dernier, en tant qu'admirateur du jardin régulier, a organisé son texte d'une façon symétrique, de telle sorte que chacun des quatre chants présente un élément du jardin (à savoir : les fleurs, les vergers, les eaux et les forêts), ce que Delille qualifie de monotone et dépourvu d'intérêt. En revanche, argumente-t-il, dans un poème consacré au "genre libre », comme dans le même jardin paysager, l'arrangement des éléments doit être le fruit de l'imagination, «naturellement amie de la liberté » et qui «regrette la beauté un peu désordonnée et la piquante irrégularité de la nature » (vii). La spécificité de la distribution des parties dans l'ensemble relèverait alors du même sujet du poème. L'auteur y revient dans une préface apparue dans les éditions postérieures de son ouvrage, où il énonce : « $[\mathrm{D}] \mathrm{ans}$ les jardins pittoresques et libres, où tous les objets sont souvent mêlés ensemble, où il a fallu remonter aux causes philosophiques du plaisir qu'excite en nous la vue de la nature embellie et non pas tourmentée par l'art, où il a fallu exclure les alignements, les distributions symétriques, les beautés compassées, un autre plan étoit nécessaire » (cf. Delille, $1863: 3$ ).

La pratique de regarder le paysage à travers le prisme de la tradition des représentations picturales fait que, dans le texte, des allusions à la peinture sont très nombreuses. Au début du chant $\mathrm{I}$, le poète écrit : «Un jardin, à mes yeux, est un vaste tableau. / Soyez peintre!»(13). Les adeptes de l'art d'embellir la nature sont invités à manipuler des éléments du paysage comparés aux outils du peintre et, en même temps, perçus en mouvement constant qui découle de la succession des jours et des nuits, des saisons et des cycles végétaux :

Les champs, leur nuances sans nombre,

Les jets de la lumière, et les masses de l'ombre,

Les heures, les saisons, variant tour-à-tour

Le cercle de l'année et le cercle du jour

${ }^{7}$ L'édition de 1782 ne mentionne pas le nom de Twickenham. Dans les éditions postérieures, Delille étend la liste de lieux admirables pour leurs jardins, paysages ou richesse végétale. Notamment, il consacre des passages assez longs aux jardins d'Angleterre et, devant la sollicitation de la comtesse Izabela Czartoryska, fait allusion à son jardin de Puławy et à celui de la princesse Helena Radziwiłłowa, à Nieborów (v. Załuska, 1934 : 28).

${ }^{8}$ Les vers « Avant tout connoissez votre site; et du lieu / Adorez le génie, et consultez le dieu » (15), de Delille, rappellent le fragment d'Epistle IV, To Richard Boyle, Earl of Burlington (1731) où Pope écrit : «Consult the Genius of the Place in all » $(1911: 258)$. 
Et des prés émaillés les riches broderies,

Et des riants côteaux les vertes draperies,

Les arbres, les rochers, et les eaux, et les fleurs,

Ce sont là vos pinceaux, vos toiles, vos couleurs.

La nature est à vous ; et votre main féconde

Dispose, pour créer des élémens du monde (13).

$\mathrm{Si}$, selon les propos déjà cités de Walpole, William Kent, en tant que concepteur du jardin paysager, s'est basé sur des principes esthétiques dérivés de la peinture, le texte de Delille s'en fait l'écho de manière très claire. On y trouve un répertoire du lexique spécifique aux arts visuels : «tableaux», « desseins », " pinceaux», « toile », « couleurs », mais aussi " peintre » ou bien « artiste des jardins » sont parsemés tout au long du poème. Delille fait également allusion à l'usage de perspective («Se déploie une immense et noble perspective», [68]) et aux différents plans de la composition (« Mille arbres [...] / Ouvroient dans le lointain une scène imprévue » [28]), utilise le clair-obscur et souligne l'importance de la distribution de couleurs et textures différentes (par exemple, des couronnes des arbres) ou du placement des groupes isolés susceptibles de focaliser l'attention du spectateur (notamment, des massifs d'arbres, si chers à Capability Brown, le paysagiste anglais le plus célèbre). Les maîtres du jardin irrégulier organisent l'espace comme s'il s'agissait d'une peinture à trois dimensions. Le poète le décrit, ainsi qu'en témoigne parfaitement ce fragment que nous citons malgré sa longueur, car il présente tous les éléments que nous venons d'évoquer :

Les bois peuvent s'offrir sous des aspects sans nombre.

Ici, des troncs pressés rembruniront leur ombre :

Là, de quelques rayons égayant ce séjour,

Formez un doux combat de la nuit et du jour.

Plus loin, marquant le sol de leurs feuilles légères,

Quelques arbres épars joueront dans la clairière [...]

Avant tout, n'allez point $[\ldots]$

Nous cacher des forêts les nombreuses familles:

Je veux le voir ; je veux, perçant au fond des bois,

Voir ces arbres divers qui croissent à la fois :

Les uns tout vigoureux et tout frais de jeunesse,

D'autres tout décrépits, tous noueux de vieillesse $[\ldots]$;

Vaste scène $[. .].(33-34)$.

Notons que les tableaux des maîtres ne sont pas, ne doivent pas être la seule source d'inspiration des jardiniers. Dans le chant IV, Delille les encourage également à suivre les pas des poètes - «Des Poètes fameux oser imiter l'art » (67). Cette nouvelle indication semble un peu moins évidente que celle de modeler le jardin à la manière d'un peintre. Les passages qui suivent proposent 
de se déplacer dans l'espace du jardin suivant l'itinéraire conseillé par le poète. De nombreuses formules rendent compte de ce mouvement : «Plus loin, c'est un beau lac $[\ldots]$ / [...] Soudain, la scène change » $(68)$; «Revenons, il est temps » (71). Suite à cette démarche, le jardin de Delille se dote d'une dimension temporelle et se présente comme une succession d'images ou de scènes qui se déroulent devant le visiteur (« Des scènes à ma voix naîtront de toutes parts » [65] ; « [l'art] d'une scène qui suit fait naître une autre scène » [66]). C'est le poète luimême qui fait allusion à cette structure, répondant à ceux qui avaient reproché à son texte le défaut de plan ${ }^{9}:$ «Le quatrième chant [...] contient la distribution des différentes scènes majestueuses ou touchantes, voluptueuses ou sévères, mélancoliques ou riantes; l'artifice avec lequel doivent être tracés les sentiers qui y conduisent » (Delille, $1863: 3$ ).

Dans le chant IV, l'espace poétique aura donc une structure épisodique, composée d'une suite de petites scènes. Il est à noter que cette disposition en scènes successives et le changement de paysage et d'ambiance qui caractérisent le projet de Delille s'adaptent parfaitement aux règles observées dans la planification - «en enfilade 》- du jardin paysager (rappelons ce que nous avons dit plus haut sur la formation d'architecte d'intérieur de Kent). En effet, dans le poème de Delille, nous retrouvons les modèles d'arrangement de l'espace employés par les praticiens du jardin paysager qui, quant à eux, s'étaient inspirés de la poésie pastorale, organisée en séries d'églogues dont chacune possède un ton et une ambiance spécifiques ${ }^{10}$; Virgile et les autres auteurs bucoliques seraient donc ces « poètes fameux » que Delille conseille d'imiter. Il propose de donner à chaque coin du jardin un caractère particulier («Inventez, hasardez des contrastes heureux $»^{11}, 69$ ), de sorte que le visiteur puisse se balader parmi des

\footnotetext{
${ }^{9}$ Voilà un fragment de la critique acerbe de Rivarol, contenue dans sa Lettre critique : «Ce cliquetis et ce désordre qui règnent avec art dans tout le Poëme, déroutent et fatiguent ses amis, qui n'ont pour se délasser qu'une continuité de préceptes, des semblants d'Épisodes, une maigreur générale et un défaut absolu d'intérêt et de mouvement. Car bien que le Poëte ait varié son méchanisme, et donné à son vers des attitudes différentes, ce n'est après tout qu'une volubilité de rithme, un mouvement intestin, et le Poëme ne marche pas : on peut le prendre et le commencer, le quitter et le reprendre à chaque page, sans que le plan et même le sens en souffrent. [...] Ils prétendent même qu'un Peintre ne pourroit jamais dessiner un tableau d'après M. D. » etc. (Barruel, $1872: 9$ et 10-11).

${ }^{10}$ « Pastoral poetry was structured so that each poem, or translated eclogue or georgic of Virgil, had its own specific tone - a moral or philosophical idea that underpinned the action. This formula translated naturally to the landscape garden as it developed, in that each garden episode, usually focused on a single future, had its own particular atmosphere or narrative context that is sustained and intensified throughout » (Richardson, $2008: 172$ ).

${ }^{11}$ C'est suite à cette recommandation que Delille mentionne le motif de « la mort dans l'Arcadie ", tout en évoquant la peinture de Poussin, dont le jardin anglais s'inspire, comme cela a déjà été dit.
} 
décors suscitant des émotions et des états d'esprit les plus variés, porteurs de sens symbolique, et en former une histoire. Chez Delille, ces émotions vont de la gaieté et du bonheur à la peine et à la douleur, représentées par les urnes et les tombeaux, en passant par la rêverie et une vague mélancolie. Les éléments architectoniques - des ruines, des restes d'un vieux fort ou une chapelle - qui rappellent les toiles de Claude Lorrain ou de Claude Gaspard, contribuent à créer une atmosphère particulière. L'expérience que vivra le visiteur, laquelle dépendra également de différentes variables et impondérables - par exemple, sa disposition psychique, le temps atmosphérique ou la saison -, doit le marquer profondément et le convaincre que le paysage qu'il a traversé est habité par un genius loci unique et inimitable.

L'apparition de l'élément temporel, rendu par la succession d'images, dans une description qui privilégie, en principe, la dimension spatiale, est un élément que l'on ne peut pas passer sous silence. Certes, elle peut être regardée, avec raison, comme un exemple d'interaction entre la littérature (art temporel) et les arts visuels (spatiaux), discutée avec beaucoup de vigueur tout au long du XVIII ${ }^{\mathrm{e}}$ siècle. Cette piste, sans aucun doute, mériterait une analyse sérieuse ; cependant, elle nous amènerait inévitablement à revenir sur un débat qu'il faudrait faire remonter à l'ut pictura poesis d'Horace ou, mieux encore, à la plus Haute Antiquité, comme nous le rappelle Praz (1970: 3), ce qui n'est pas l'objectif de notre article. Par conséquent, nous voulons seulement souligner que, dans le texte de Dellile, l'introduction du facteur temporel passe indubitablement par l'imitation du modèle de jardin irrégulier. En d'autres termes, grâce à la succession propre du jardin irrégulier - une succession issue, pour sa part, de la poésie pastorale -, Delille réussit à introduire dans sa descriptio le facteur temporel. Cette démarche, qui relève directement des propos traités, c'est-à-dire de la présentation du modèle de jardin doté d'une dimension temporelle, distingue l'ouvrage de Delille des autres poèmes descriptifs mentionnés au début de l'article. C'est la raison pour laquelle nous voulons insister, une fois de plus, sur le fait que toute analyse approfondie des textes doit prendre en compte leur spécificité thématique.

Pour terminer, nous revenons sur les analyses critiques consacrées aux Jardins. Dans son «portrait littéraire» de Delille, Sainte-Beuve écrivait, en citant Jean-François La Harpe :

L'auteur de l'Année littéraire [...] prononçait que le poëme de l'abbé Delille était un véritable jardin anglais : «On pourrait, dit-il, être tenté de croire que le poëme est construit de morceaux détachés et de pièces de rapport réunies sous le même titre. Les idées y semblent jetées au hasard, déchiquetées par petits couplets qu'étrangle à la fin une sentence ». Ce reproche est fondamental à l'égard de Delille et tient à la nature même de son procédé (Sainte-Beuve, $1884: 82$ ). 
Il est facile de remarquer que les deux critiques perçoivent le «jardin anglais » comme un modèle négatif, celui du désordre : le poème s'y adapte et c'est la raison pour laquelle sa structure se révélerait discontinue et chaotique. Dans ce très court aperçu consacré à la tradition paysagiste dans les Jardins de Delille, nous avons adopté un point de vue contraire, en considérant le jardin irrégulier comme une manière d'ordonner des éléments dans l'espace. Cela nous a permis, tout d'abord, d'apercevoir la logique qui organise l'espace du jardin chez Delille et fonde le déploiement du sens dans son discours. Il est à noter également que la thématique abordée dans le poème explique l'apparition des références à la poésie et à la peinture qui, bien loin d'être des ornements superflus, se constituent comme des supports importants du discours. Grâce à ces références, «le poème sur le jardin à l'anglaise » peut se révéler comme une modalité très particulière du poème descriptif sur la nature. Non seulement il présente un cas d'une exceptionnelle densification des rapports entre la littérature et la peinture, mais il porte surtout une réflexion sur les liens et les écarts entre la réalité et sa représentation artistique. 


\section{BIBLIOGRAPHIE}

BARRUel, Le COMTE DE (1782). Lettres critiques sur le Poème des Jardins, suivies du Chou et du Navet. Amsterdam et Paris : Chez les Marchands de Nouveautés.

Barthes, Roland (1970). S/Z. Paris : Seuil.

CASTEl ReNE-RichaRd (1802). Les plantes, poëme. $3^{\mathrm{e}}$ éd. Paris : Deterville.

Chenier, MARIE-Joseph (1824). CEuvres posthumes. Vol. 3. Paris : Guillaume.

DE BRUYN, ODILE (2001). "Les promoteurs du jardin paysager "à l'anglaise" au XVIII siècle et les jardins gréco-romains... en quête de légitimité et de modèles ?». Revue belge de philologie et d'histoire 79, fasc. 1 : 127-169.

Delille, JACQues (1782). Les Jardins ou l'art d'embellir les paysages. Poème. Paris : Valade et Cazin.

- (1863). Euvres complètes. $5^{\mathrm{e}}$ éd. Paris : Fermin Didot.

MAYER, LAURA (2011). Capability Brown and the English landscape garden. London: Shire Publications.

Pope, AleXANDER (1911). The poetical works. London : Macmillan et Co.

Praz, Mario (1974). Mnemosyne. The Parallel Between Literature and the Visual Arts. Princeton : Princeton University Press.

RichARDSON, TIM (2008). The Arcadian Friends. Inventing the English landscape garden. London et al. : Bantam Press.

Sainte-Beuve, Charles-Augustin (1884). Portraits littéraires. Vol 2. Paris : Garnier Frères.

Saint-Lambert, Jean-François (1796). Les Saisons. Poëme. Paris : P. Didot l'aîné.

Villanueva, Darío (1997). Theories of Literary Realism. Trad. Mihai I. Spariosu et S. GarcíaCastañon. Albany : State University of New York Press.

Walpole, Horatio (1798). The Works. Vol. II. London : G. G. et J. Robinson et al.

ZaŁuska, Apolonja (1934). Poezja opisowa Delille’a w Polsce. Kraków : Skład Główny w Kasie im. J. Mianowskiego. 